\title{
A two-dimensional model of the deformation of photoresist structures using elastoplastic polymer properties
}

\author{
K. Yoshimoto, M. P. Stoykovich, H. B. Cao, ${ }^{\text {a) }}$ J. J. de Pablo, and P. F. Nealey ${ }^{\text {b) }}$ \\ Department of Chemical Engineering and Center for Nanotechnology, University of Wisconsin, \\ 1415 Engineering Drive, Madison, Wisconsin 53706 \\ W. J. Drugan \\ Department of Engineering Physics, University of Wisconsin, 1500 Engineering Drive, Madison, \\ Wisconsin 53706
}

(Received 2 February 2004; accepted 12 May 2004)

\begin{abstract}
A model was developed for predicting the collapse behavior of photoresist structures due to the drying of rinse liquids during wet chemical processing. The magnitude of the capillary forces was estimated using the classical thermodynamics of surface tension, and the deformation of the structure was modeled using beam bending mechanics that accounts for both elastic and plastic modes of deformation. The two-dimensional model can predict the critical beam height of collapse as a function of the wetting behavior of the rinse liquid on the beam, the elastic and plastic mechanical properties of the polymeric photoresist, and the beam dimensions. Collapse behavior was predicted for polymer nanostructures with elastoplastic mechanical properties similar to those of bulk poly(methyl methacrylate). We have compared the collapse predictions from our model with the results of models that account only for elastic or plastic deformation behavior. Regimes in the elastic-plastic mechanical property space for which it is necessary to use the developed beam bending model have been highlighted. It is shown that in some cases the inclusion of both elastic and plastic mechanical properties is necessary for modeling the collapse behavior of polymer beams fabricated using the lithographic process. () 2004 American Institute of Physics.
\end{abstract}

[DOI: $10.1063 / 1.1768614]$

\section{INTRODUCTION}

The semiconductor industry is constantly pushing the limits of the lithographic process used to fabricate integrated circuits. Structures are routinely generated with at least one dimension less than $150 \mathrm{~nm}$, and the smallest features have a minimum dimension, known as the critical dimension, of approximately $70 \mathrm{~nm} .{ }^{1}$ The basic lithographic process used to fabricate microscopic and nanoscopic systems involves spinning a film of radiation-sensitive polymeric photoresist onto a substrate, followed by an exposure step and sometimes a bake step to induce dissolution rate differences between the exposed and unexposed regions. ${ }^{2}$ A development step followed by liquid rinsing and drying processes are used to produce the desired pattern in the photoresist. This photoresist pattern can subsequently be transferred to the substrate via etching techniques. The International Technology Roadmap for Semiconductors predicts that the patterned photoresist features must have an aspect ratio, defined as the ratio of feature height to critical dimension, of at least 3 to 4 to provide the necessary level of etch resistance for all future technology nodes. ${ }^{1}$ However, an emerging difficulty in the fabrication of densely packed, high aspect ratio structures using standard polymeric photoresists is that of pattern collapse. Pattern collapse is the deformation of the photoresist

\footnotetext{
${ }^{a)}$ Present address: Intel Corporation, RA1-240, 5200 N.E. Elam Young Parkway, Hillsboro, OR 97124.

b) Author to whom correspondence should be addressed; electronic mail: nealey@engr.wisc.edu
}

structures in response to unbalanced capillary forces present during the drying step of the lithographic process. ${ }^{3}$ To understand the collapse phenomenon it is necessary to develop appropriate predictive models. Tanaka et al. utilized classical beam bending equations and the classical thermodynamics of surface tension to predict collapse behavior based on the structure's dimensions and the material's elastic properties. ${ }^{4}$ The elastic mechanical properties of photoresists have been determined by comparing the model predictions with the collapse of experimental structures.

Past work by our group suggests that photoresist structures undergo both elastic and plastic deformation during pattern collapse. ${ }^{5}$ Deformed structures which display plastic yielding near the substrate-photoresist interface have been observed in scanning electron microscopy (SEM) images of collapse (see Fig. 1). ${ }^{5}$ In addition, we have developed nanoscale test structures of photoresist beams that deform in response to carefully well-defined capillary forces; ${ }^{6}$ by comparing the bending behavior of one- and two-beam test structures it has been observed that plastic yielding may occur even for small deformations. The designed test structures, when employed in concert with appropriate beam bending models, should also provide a powerful technique for measuring the mechanical properties of photoresists. The developed beam bending model must consider both the elastic and plastic deformation behavior of polymeric photoresists. The beam bending model developed by Tanaka et al. considers only elastic mechanical properties and hence ignores the yielding and plastic deformation observed in poly- 


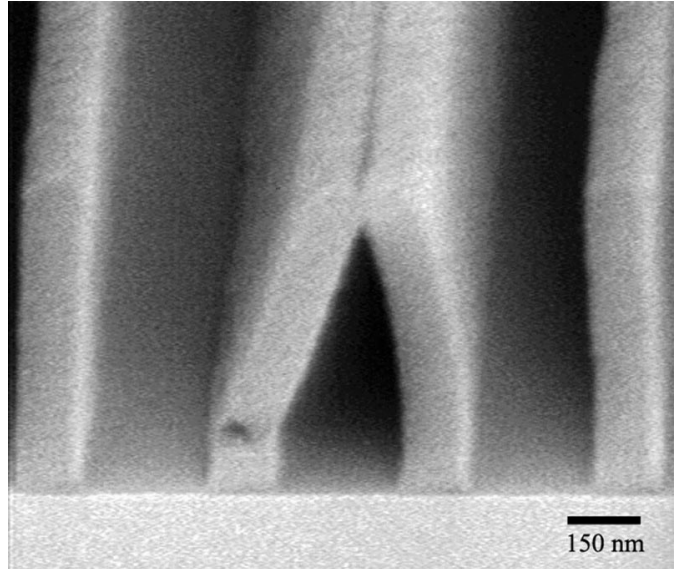

FIG. 1. Cross-sectional scanning electron micrograph of collapsed Apex E photoresist structures. The pattern consists of dense lines $150 \mathrm{~nm}$ wide with a period of $400 \mathrm{~nm}$.

mers, especially glassy polymers, at relatively small strains. ${ }^{4}$ Alternative models of collapse have assumed a purely rigidplastic response and should only be applied to stiff materials in the limit of extremely narrow beams. ${ }^{7}$

In this paper we develop a continuum-level beam bending model that incorporates both the elastic and plastic mechanical properties observed for polymer materials such as photoresists. It is demonstrated that when combined with a model of the capillary forces, the elastoplastic beam bending model can be used to predict the critical dimensions of pattern collapse for photoresist structures. A comparison between our elastoplastic beam bending model and the solely elastic and solely plastic models is performed, and the mechanical property regimes in which the deformation models become different are discussed.

\section{TWO-DIMENSIONAL MODELS OF ELASTIC AND PLASTIC BEAM BENDING}

The models discussed in this paper have been applied to systems in which two parallel polymer structures of width $W$ are separated by a narrow space of dimension $d$ that is filled with rinse liquid. On the outside of the beams it is assumed that there is no rinse liquid. Figure 2(a) depicts the threedimensional situation of interest. This complex threedimensional case can be simplified to a two-dimensional model if it is assumed that the structures have a much greater length $D$ than separation $d$ and in-plane height and width $H$ and $W$, respectively. The capillary forces present in this system require that the beams deform towards each other [see Fig. 2(b)], and collapse is said to occur if the beams remain permanently deformed or are touching after completion of the drying process.

Any model of photoresist deformation during the drying process must consider both the capillary forces acting on the structures and the deformation behavior of the beams in response to the imposed force. The capillary forces present during the drying process may be estimated by the YoungLaplace equation in two dimensions. ${ }^{8}$ The pressure difference across the rinse liquid and air interface, $\Delta P$, is

$$
\Delta P=\gamma\left(\frac{1}{r_{1}}+\frac{1}{r_{2}}\right)
$$

where $\gamma$ is the rinse liquid surface tension, and $r_{1}$ and $r_{2}$ are the principal radii of curvature of the meniscus perpendicular and parallel to the structures, respectively. If it is assumed that the pressure above the meniscus and on the outside of the polymer features is at atmospheric conditions, then the quantity $\Delta P$ also represents the difference in pressure acting on the outside and inside walls of the beams. The net capillary force $F$ acting on the outside surface of each polymer structure is

$$
F=\Delta P H D
$$

where $H$ and $D$ represent the height and depth of the structure, respectively.

The radii of curvature of the meniscus can be estimated by modeling the shape of the liquid surface as a section of a cylinder [see Fig. 2(a)] and assuming a constant receding contact angle $\theta$ of the rinse liquid on the polymer. The radii of curvature are estimated as a)

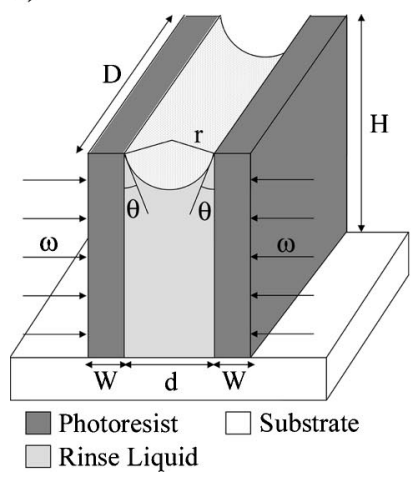

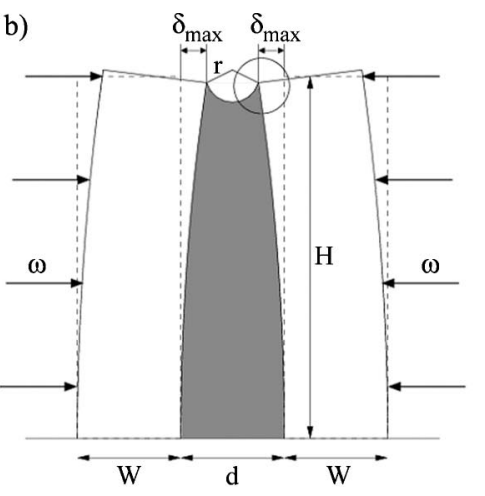

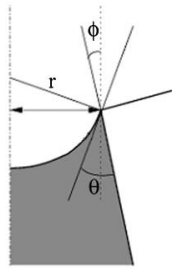

FIG. 2. Geometry examined by the two-dimensional beam bending models. In each model the rinse liquid is trapped between two parallel polymer beams, while the outside pools are assumed to be very large and do not contain any rinse liquid. (a) A three-dimensional view of the polymer beam geometry prior to deformation indicates how the rinse liquid meniscus can be modeled as a portion of a cylindrical surface. The beams are initially separated by a distance $d$ and each beam has a width $W$, a height $H$, and a length $D$. The initial capillary line force $\omega$ acting on the polymer beams is a function of the meniscus' radius of curvature $r$, the receding contact angle $\theta$, and the rinse liquid surface tension $\gamma$. (b) The two-dimensional cross-section view of the beams during deformation demonstrates that the meniscus' radius of curvature is dependent upon the maximum deflection $\delta_{\max }$ and the angle of deflection $\phi$ of the beams. 


$$
\begin{aligned}
& r_{1}=d / 2 \cos \theta \text { and } \\
& r_{2}=\infty
\end{aligned}
$$

when $D \gg d$. Consequently, the line force $\omega$ felt by each structure can be calculated by combining Eqs. (1)-(3),

$$
\omega=\frac{F}{H}=\Delta P D=\frac{2 \gamma D \cos \theta}{d} .
$$

It is important to note that Eq. (4) considers only the initial capillary forces and does not include the increase in forces which occurs as the structures deform and become closer together at their tops. As demonstrated in Fig. 2(b), the meniscus' radius of curvature is decreased as the beams deform. Incorporation of details on the maximum displacement $\delta_{\max }$ and the angle of deflection $\phi$ of the structure allows the radius of curvature to be determined throughout the deformation process,

$$
r=\frac{d-2 \delta_{\max }}{2 \cos (\theta-\phi)} .
$$

Substituting Eq. (5) into Eq. (4) allows the line force

$$
\omega=\frac{2 \gamma D \cos (\theta-\phi)}{d-2 \delta_{\max }}
$$

to be calculated throughout the deformation of the structure.

In previous work beam bending models based on either purely elastic or purely plastic material properties have been used to characterize nanoscale deformation behavior. Tanaka et al. developed a model for the deformation of photoresist structures involving perfectly elastic cantilevers. ${ }^{4}$ Using classical continuum-level mechanics the displacement of the top of the photoresist structure is given by

$$
\delta_{\max }=\frac{\omega H^{4}}{8 E I}
$$

and $\phi$ is given by

$$
\tan \phi=\frac{\omega H^{3}}{6 E I}
$$

in which $E$ is the Young's modulus of the elastic material and $I$ is the moment of inertia. ${ }^{9}$ For the beam geometry $I$ is defined as

$$
I=\frac{D W^{3}}{12} .
$$

It is important to note that Eqs. (7) and (8) apply only in the elastic regime and cannot appropriately be applied to beams undergoing plastic deformation. Tanaka et al. obtained an expression for $\delta_{\max }$ by substituting the line force [Eq. (6)] into Eq. (7), ${ }^{4}$

$$
\begin{aligned}
\delta_{\max }= & \left(\frac{d}{4}-\frac{\gamma H^{3} \sin \theta}{E W^{3}}\right) \\
& -\sqrt{\left(\frac{d}{4}-\frac{\gamma H^{3} \sin \theta}{E W^{3}}\right)^{2}-\frac{3 \gamma H^{4} \cos \theta}{2 E W^{3}}} .
\end{aligned}
$$

The critical structure height of collapse, $H_{c}$, can be calculated by requiring that the slope $d \delta_{\max } / d H$ goes to infinity when collapse occurs. The physical interpretation of this collapse criterion is that when $d \delta_{\max } / d H \rightarrow \infty$, the elastic restoring force of the bent elastic beam is unable to balance the increasing capillary forces and the structures will continue to be displaced until they come into contact. The resulting expression for $H_{c}$ was the cubic equation

$$
H_{c}^{3}+H_{c}^{2} \sqrt{\frac{3 E W^{3} \cos \theta}{2 \gamma \sin ^{2} \theta}}-\frac{E W^{3} d}{4 \gamma \sin \theta}=0 .
$$

Consequently, the critical collapse height could be determined when the structure dimensions ( $W$ and $d$ ), the physical rinse liquid properties $(\theta$ and $\gamma$ ), and the elastic mechanical property $(E)$ were specified.

A deformation model based solely on the yield stress $\sigma_{Y}$ of the material has been utilized by Namatsu et al. to examine the collapse of narrow $\mathrm{Si}$ structures. ${ }^{7}$ When an initial capillary line force is applied to the beams, the point of maximum stress $\sigma_{\max }$ occurs at the corners of the structuresubstrate interface. The maximum stress was given by $^{9}$

$$
\sigma_{\max }=\frac{\omega H^{2} W}{4 I}
$$

and collapse was assumed to occur when $\sigma_{\max }$ equaled or exceeded the yield stress of the material. Applying these assumptions, $H_{c}$ was determined to be

$$
H_{c}=W \sqrt{\frac{\sigma_{Y} d}{6 \gamma \cos \theta}} .
$$

Note that this expression does not include any information on the material's elastic behavior, nor does it account for the increase in capillary forces that occurs during elastic deformation. This plastic beam bending model should not be applied to polymeric microstructures because complete structure collapse cannot be equated with mechanical yielding at a single point within the structure. Such a plastic model is physically relevant only in the limit of extremely narrow beams of stiff and brittle materials.

These continuum-level mechanics models represent the divergent limits of microstructure deformation. The work of Tanaka et al. assumes perfect elastic deformation, while Namatsu et al. have determined pattern collapse based solely on the yield stress of the material. The perfectly elastic deformation model was developed for photoresist materials patterned using x-ray radiation. ${ }^{4}$ Application of the model to a polymeric photoresist provided an estimate for the Young's modulus that matched closely with that of the resist's novolak base resin. In this paper we show that there are regimes of deformation that can be sufficiently modeled using only elastic material properties; however, this simplified elastic model cannot appropriately be applied to materials that undergo significant plastic yielding during deformation. In comparison, Namatsu and co-workers have applied their model to the deformation of narrow, parallel silicon beams fabricated using electron beam lithography and chemical etching techniques. ${ }^{7}$ Although successfully applied to thin beams of silicon, this model remains valid only for stiff or high moduli materials which do not undergo any consider- 


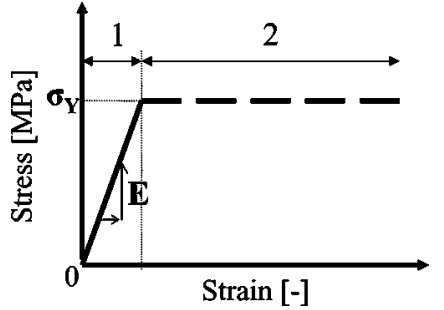

FIG. 3. The simplified stress-strain behavior of the elastoplastic model. The elastoplastic model reduces the complex stress-strain behavior observed for polymers to an elastic regime at low strains (region 1 -solid line) characterized by the Young's modulus $E$ and a perfectly plastic regime at larger strains (region 2-dashed line) characterized by a constant yield stress, $\sigma_{Y}$.

able elastic deformation prior to collapse. It cannot be applied to photoresist or polymer structures that deform both elastically and plastically at small strains.

\section{TWO-DIMENSIONAL ELASTOPLASTIC BEAM BENDING MODEL}

The following is a beam bending model for polymer microstructures and nanostructures that accounts for the elastic and plastic mechanical behavior of polymer materials. The complicated stress-strain curve observed in glassy polymers has been idealized by the elastic-perfectly plastic (elastoplastic) model of Fig. 3. In this simplified model the linear elastic regime is characterized by the Young's modulus $E$ and the perfectly plastic regime by a constant yield stress $\sigma_{Y}$ for all strains. ${ }^{9}$ Note that the viscoelastic behavior of glassy polymers is ignored by this simplification.

Stresses are induced within each structure as the beams begin to deform elastically in response to the capillary forces. The expected stress distribution near the fixed base of the structure has been illustrated for different stages of deformation (see Fig. 4); the arrows in Fig. 4 represent the normal stresses experienced during the beam bending process. As expected, the regions of maximum stress are located
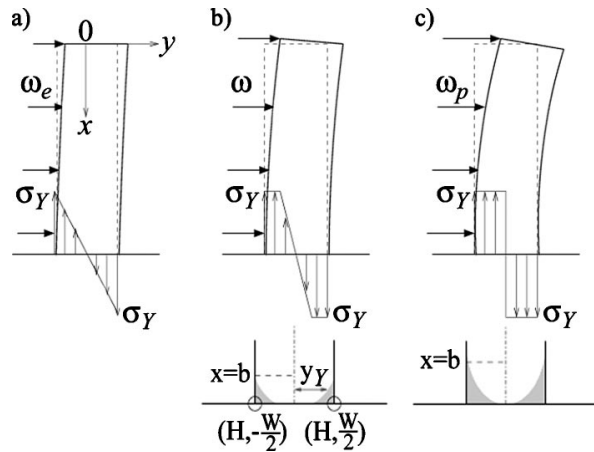

FIG. 4. The normal stress distributions at the base of the beam at different stages of the deformation process. The relative magnitudes of the stresses are represented by the lengths of the arrows. (a) The corners of the beam begin to undergo plastic deformation when the stress reaches $\sigma_{Y}$ at an applied line force of $\omega_{e}$. (b) Greater portions of the beam will yield and undergo plastic deformation as the applied line force is further increased. (c) The entire beam will eventually undergo plastic deformation when the line force reaches $\omega_{p}$. The shaded areas at the bottom of (b) and (c) represent the regions of the beam that are yielding plastically. at the corners of the structure. The normal stress profile throughout the structure when the response is purely elastic is given by

$$
\sigma(x, y)=\frac{M y}{I}
$$

in which the bending moment $M$ is

$$
M=\frac{\omega x^{2}}{2}
$$

and $x$ and $y$ are the coordinates normal and parallel to the substrate, respectively. At the corner at which $y=W / 2$ and $x=H$, the normal stress [Eq. (14)] can be simplified to Eq. (12). If the applied line force is large enough to cause the stress at the corners to equal the material's yield stress, then the corners will begin to undergo plastic deformation [see Fig. 4(a)]. Plastic deformation of the structure is initiated when the line force equals

$$
\omega_{e}=\frac{4 \sigma_{Y} I}{H^{2} W}=\frac{\sigma_{Y} D W^{2}}{3 H^{2}} .
$$

Larger portions of the structure will deform plastically as the applied line force is further increased [see Fig. 4(b)]. The normal stress profile will equal the material's yield stress at the outside of the structure, while the center region of the structure still undergoes elastic deformation and has a sloped stress profile. The boundary $b$ between elastic and plastic deformation in the $x$ direction at $y=W / 2$ is determined by equating the normal stress to the yield stress,

$$
b=2 \sqrt{\frac{\sigma_{Y} I}{\omega W}}=W \sqrt{\frac{\sigma_{Y} D}{3 \omega}} .
$$

The bending moment from $x=b$ to $x=H$ is calculated from the normal stress profile and is given by

$$
\begin{aligned}
M_{e p} & =2 \int_{0}^{y_{Y}} y\left\{\frac{\sigma_{Y}}{y_{Y}} y D d y\right\}+2 \int_{y_{Y}}^{W / 2} y\left(\sigma_{Y} D d y\right) \\
& =D \sigma_{Y}\left(\frac{W^{2}}{4}-\frac{y_{Y}^{2}}{3}\right)
\end{aligned}
$$

where $y_{Y}(\geqslant 0)$ is the $y$ component of the border between the elastic and plastic regions. An expression for $y_{Y}$ as a function of $x(b \leqslant x \leqslant H)$ is obtained by equating Eqs. (15) and (18),

$$
y_{Y}=\sqrt{3\left(\frac{W^{2}}{4}-\frac{\omega x^{2}}{2 \sigma_{Y} D}\right)} .
$$

As the applied line force is further increased, the material across the entire structure's base will eventually undergo plastic deformation [see Fig. 4(c)]. The minimum line force for complete plastic deformation is given by

$$
\omega_{p}=\frac{\sigma_{Y} D W^{2}}{2 H^{2}} .
$$

If a line force larger than $\omega_{p}$ is applied to the structure, the material can no longer impede deformation and complete collapse will occur. 
Equations (14) through (20) present a means for calculating the normal stress distribution throughout the base of the structures; however, it is also important to calculate the maximum displacement of the features. A material undergoing plastic deformation offers no resistance to its displacement in response to the imposed line force. Therefore if any portion of the material at the base of the structure deforms plastically, the maximum displacement of the top of the structure is greater than that calculated for purely elastic deformation. The bending moment and moment of inertia in the material undergoing plastic deformation $[b \leqslant x \leqslant H$ of Figs. 4(b) and 4(c)] are

$$
M_{e p}=2 \int_{0}^{y_{Y}} y\left\{\frac{\sigma_{Y}}{y_{Y}} y D d y\right\}=\frac{2 \sigma_{Y} D y_{Y}^{2}}{3}
$$

and

$$
I_{e p}=\int_{0}^{D} \int_{-y_{Y}}^{y_{Y}} y^{2} d y d z=\frac{2 D y_{Y}^{3}}{3},
$$

respectively. The curvature of the neutral surface in the plastically deforming region can be calculated from Eqs. (21) and (22),

$$
\frac{d^{2} y}{d x^{2}}=\frac{M}{E I}=-\frac{\sigma_{Y}}{E y_{Y}} .
$$

Similarly, the curvature of the neutral surface in the elastically deforming region can be determined from Eqs. (9) and (14),

$$
\frac{d^{2} y}{d x^{2}}=\frac{M}{E I}=-\frac{6 \omega x^{2}}{E D W^{3}} .
$$

The displacement $\delta_{\max }$ and angle $\phi$ of the top of the deforming structure can be calculated by integrating Eqs. (23) and (24) with the following boundary conditions: (1) continuity between the elastically and plastically deforming regions and (2) no deformation at the base of the structure $\left(\delta_{x=H}=0\right.$ and $\phi_{x=H}=0$ ),

$$
\begin{aligned}
\delta_{\max }= & \frac{3 \omega b^{4}}{2 E D W^{3}}+\frac{\sigma_{Y}}{E} \sqrt{\frac{2 \sigma_{Y} D}{3 \omega}}\left(\sqrt{\frac{\sigma_{Y} D W^{2}}{2 \omega}-b^{2}}\right. \\
& \left.-\sqrt{\frac{\sigma_{Y} D W^{2}}{2 \omega}-H^{2}}\right), \\
\tan \phi= & \frac{2 \omega b^{3}}{E D W^{3}}-\frac{\sigma_{Y}}{E} \sqrt{\frac{2 \sigma_{Y} D}{3 \omega}}\left\{\sin ^{-1}\left(\frac{b}{W} \sqrt{\frac{2 \omega}{\sigma_{Y} D}}\right)\right. \\
& \left.-\sin ^{-1}\left(\frac{H}{W} \sqrt{\frac{2 \omega}{\sigma_{Y} D}}\right)\right\} .
\end{aligned}
$$

The variables $b$ and $H$ can be eliminated by substituting Eqs. (17) and (20),

$$
\begin{aligned}
\delta_{\max }= & \frac{\sigma_{Y}^{2} D W}{E \omega}\left\{\frac{1}{2}-\sqrt{\frac{1}{3}\left(1-\frac{\omega}{\omega_{p}}\right)}\right\}, \\
\tan \phi= & \frac{\sigma_{Y}}{E} \sqrt{\frac{2 \sigma_{Y} D}{3 \omega}}\left\{\frac{\sqrt{2}}{3}-\sin ^{-1}\left(\sqrt{\frac{2}{3}}\right)\right. \\
& \left.+\sin ^{-1}\left(\sqrt{\frac{\omega}{\omega_{p}}}\right)\right\} .
\end{aligned}
$$

It can be demonstrated that Eqs. (27) and (28) are reduced to the expressions that describe purely elastic deformation [Eqs. (7) and (8)] when $\omega=\omega_{e}=2 \omega_{p} / 3$.

The developed two-dimensional elastoplastic beam bending model is used to calculate the onset of collapse by following an approach similar to that of Tanaka et al..$^{4}$ Equations (6), (27), and (28) have been combined to account for the increase in applied capillary forces that occurs during deformation. Assuming that only small deformations occur results in

$$
\begin{aligned}
\omega d & -\frac{2 \sigma_{Y}^{2} D W}{E}\left\{\frac{1}{2}-\sqrt{\frac{1}{3}\left(1-\frac{\omega}{\omega_{p}}\right)}\right\} \\
& =2 \gamma D\left[\cos \theta+\frac{\sigma_{Y} \sin \theta}{E} \sqrt{\frac{2 \sigma_{Y} D}{3 \omega}}\left\{\frac{\sqrt{2}}{3}\right.\right. \\
& \left.\left.-\sin ^{-1}\left(\sqrt{\frac{2}{3}}\right)+\sin ^{-1}\left(\sqrt{\frac{\omega}{\omega_{p}}}\right)\right\}\right] .
\end{aligned}
$$

This expression, similar in some aspects to Eq. (10), can be solved for the critical conditions of collapse. A new parameter $\alpha$ defined as

$$
\alpha=\frac{\omega}{\omega_{p}}
$$

is introduced to simplify the solution. In the elastoplastic model the line force can range between $\omega=\omega_{e}=\frac{2}{3} \omega_{p}$ and $\omega$ $=\omega_{p}$; therefore for collapse it must hold that

$$
\frac{2}{3} \leqslant \alpha \leqslant 1 \text {. }
$$

Equation (29) can thus be simplified to a cubic equation with respect to $\omega$,

$$
\omega^{3}-2 R \omega^{2}+R^{2} \omega-S^{2}=0,
$$

where

$$
R(\alpha)=\frac{2}{d}\left\{\frac{\sigma_{Y}^{2} D W}{E}\left(\frac{1}{2}-\sqrt{\frac{1-\alpha}{3}}\right)+\gamma D \cos \theta\right\}
$$

and

$$
\begin{aligned}
S(\alpha)= & \frac{2 \gamma \sigma_{Y} D \sin \theta}{d E} \sqrt{\frac{2 \sigma_{Y} D}{3}}\left\{\frac{\sqrt{2}}{3}-\sin ^{-1}\left(\sqrt{\frac{2}{3}}\right)\right. \\
& \left.+\sin ^{-1}(\sqrt{\alpha})\right\} .
\end{aligned}
$$

This equation, unlike Eq. (11), cannot be solved explicitly for the critical height of collapse and must instead be solved numerically. Equation (32) can be solved for $\omega$ for a given $\alpha$ within the appropriate range [Eq. (31)]. Values for $H(\alpha)$ and 
a)

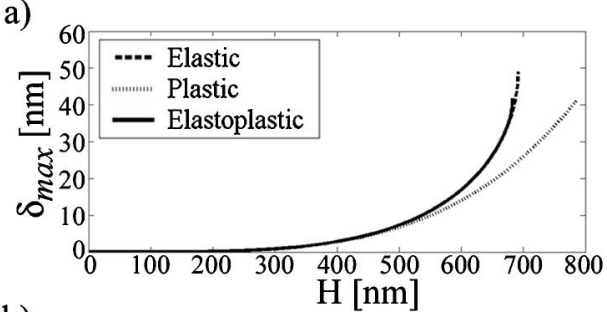

b)

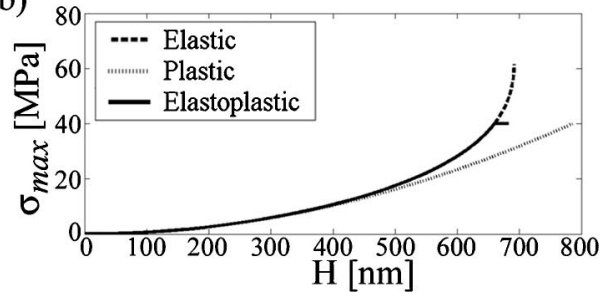

c)
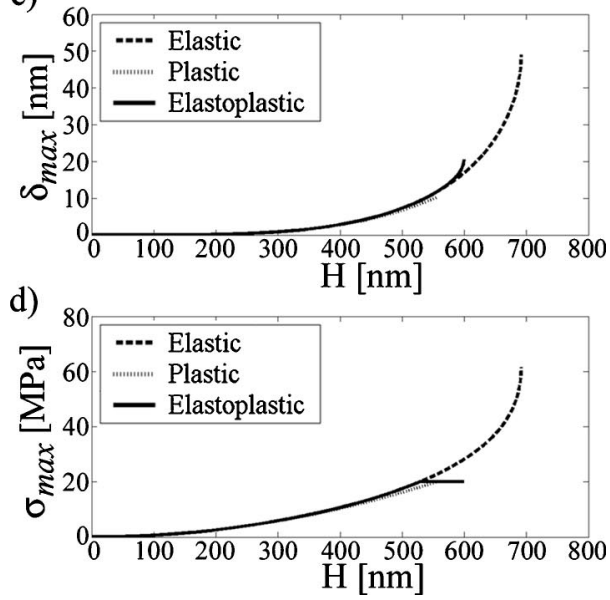

FIG. 5. Comparison of the beam bending models that consider elastic, plastic, and elastoplastic mechanical properties. Plots of (a) $\delta_{\max }$ and (b) $\sigma_{\max }$ as a function of beam height $H$ have been calculated for the case of $E=3 \mathrm{GPa}$ and $\sigma_{Y}=40 \mathrm{MPa}$. Also included are (c) $\delta_{\max }$ and (d) $\sigma_{\max }$ versus $H$ for the case of $E=3 \mathrm{GPa}$ and $\sigma_{Y}=20 \mathrm{MPa}$. Both sets of plots have been constructed using the parameters $d=200 \mathrm{~nm}, W$ $=100 \mathrm{~nm}, \gamma=21.7 \mathrm{mN} / \mathrm{m}$, and $\theta=5^{\circ}$. $\delta_{\max }(\alpha)$ can then be calculated from Eqs. (20) and (27), respectively. However, structure collapse occurs only if the elastic restoring force of the material is insufficient to balance the deformation forces and $d \delta_{\max } / d H \rightarrow \infty$. It is possible to check the critical collapse condition

$$
\frac{\delta_{\max }(\alpha+\Delta \alpha)-\delta_{\max }(\alpha)}{H(\alpha+\Delta \alpha)-H(\alpha)} \rightarrow \infty
$$

by starting with an initial guess of $\alpha=2 / 3$ and increasing $\alpha$ in small increments. Eventually the proper $\alpha$ value is determined, Eq. (35) is satisfied, and the critical height of collapse is calculated.

\section{RESULTS AND DISCUSSION}

In our elastoplastic beam bending model there are six independent variables that must be specified in order to solve for the critical structure dimensions at collapse. These variables include the physical properties of the rinse liquid $\theta$ and $\gamma$, the structure's mechanical properties $E$ and $\sigma_{Y}$, and two out of the three structure dimensions $W, H$, and $d$. In this paper fixed values of $W$ and $d$ have been assumed due to the mathematical complexity of Eq. (32), and the critical height has been numerically calculated. For most of this section we consider the case of $d=200 \mathrm{~nm}, W=100 \mathrm{~nm}, \quad \gamma$ $=21.7 \mathrm{mN} / \mathrm{m}$, and $\theta=5^{\circ}$. The examined mechanical properties of $E=3 \mathrm{GPa}$ and $\sigma_{Y}=20-40 \mathrm{MPa}$ are reasonable values for many glassy polymers and photoresists. ${ }^{10,11}$

A comparison between our elastoplastic model, the elastic model of Tanaka et al., and the plastic model of Namatsu et al. is performed in Fig. 5. The maximum structural deflection and stress are plotted as a function of structure height in Figs. 5(a) and 5(b), respectively, for the case of $E=3 \mathrm{GPa}$ and $\sigma_{Y}=40 \mathrm{MPa}$. For the elastic model, $\delta_{\max }$ increases until the slope $d \delta_{\max } / d H$ becomes infinite at a critical height of $692 \mathrm{~nm}$. Although the stress is not necessarily required for the elastic model, $\sigma_{\max }$ can be calculated by

$$
\sigma_{\max }=\frac{2 E W}{H^{2}} \delta_{\max }
$$

as derived from Eqs. (7) and (12). The maximum stress curve in Fig. 5(b) is also shown to have an infinite slope at the critical height. It is important to note that at the critical height $\sigma_{\max }$ calculated by the elastic model is greater than $\sigma_{Y}$ of the material. This physically unrealistic situation suggests that the accuracy of the elastic model depends on the magnitude of the yield stress of the material.

The plastic model assumes that structure collapse can be calculated based simply on the initial line force. Equation (12) is used to evaluate $\sigma_{\max }$, and $\delta_{\max }$ is quantified from Eq. (7). The critical height for the plastic model is determined by equating $\sigma_{\max }$ to $\sigma_{Y}$. For the given parameters the critical height is calculated to be $785 \mathrm{~nm}$ and is much larger than $H_{c}$ predicted by the elastic and elastoplastic models [see Fig. 5(a)]. The plastic model generally overestimates the critical height for collapse of materials that have large yield stresses. This overestimation results from ignoring the increase in capillary forces acting on the structures during deformation.

The results of Figs. 5(a) and 5(b) indicate that for materials with a high yield stress the elastoplastic model predicts similar deformation behavior to the elastic model. The critical height predicted by the elastoplastic model is $683 \mathrm{~nm}$, only $9 \mathrm{~nm}$ or $1.3 \%$ smaller than the elastic model prediction. At small values of $H$ the entire structure only undergoes elastic deformation $\left(\sigma_{\max }<\sigma_{Y}\right)$, and the elastoplastic and elastic models predict the same maximum displacements and stresses. Figure 5(b) demonstrates that the models diverge as the capillary forces experienced by the structures increase and cause some of the material to deform plastically $\left(\sigma_{\max }\right.$ $\left.=\sigma_{Y}\right)$. In contrast to the elastic model, the elastoplastic model properly accounts for the plastically deforming regions that offer no resistance to displacement of the structure. However, the case of $E=3 \mathrm{GPa}$ and $\sigma_{Y}=40 \mathrm{MPa}$ demonstrates that there are some regimes in $E$ and $\sigma_{Y}$ parameter space for which the elastic and elastoplastic beam bending models predict nearly identical critical heights of collapse.

To demonstrate the importance of incorporating both elastic and plastic deformation into a beam bending model a material having the mechanical properties of $E=3 \mathrm{GPa}$ and $\sigma_{Y}=20 \mathrm{MPa}$ is examined in Figs. 5(c) and 5(d). Since the elastic model does not account for plastic yielding, changing the yield stress does not influence the elastic model calculations and the critical height of collapse is again predicted to be $692 \mathrm{~nm}$. Similarly the elastic and elastoplastic models predict the same deformation behavior until the maximum stress reaches the yield stress of the material. It is found in 
a)

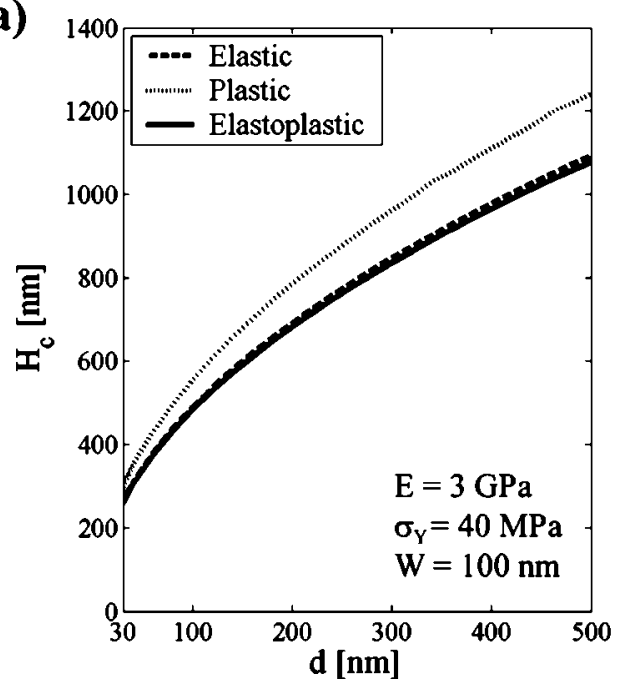

b)

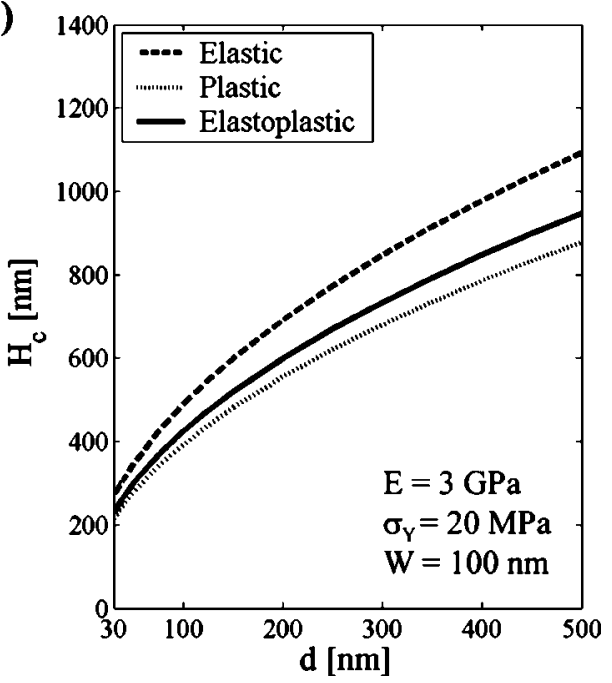

FIG. 6. Comparison between the critical heights of collapse predicted by the elastic, plastic, and elastoplastic models as a function of the beam separation $d$. (a) When $E=3 \mathrm{GPa}$ and $\sigma_{Y}=40 \mathrm{MPa}$ the $H_{c}$ estimated by the elastic and elastoplastic models are nearly equivalent for the entire range of $d$. (b) In the case of low yield stresses such as $E=3 \mathrm{GPa}$ and $\sigma_{Y}=20 \mathrm{MPa}$ the $H_{c}$ predictions of the elastic and elastoplastic models become significantly different. Both plots utilize $W=100 \mathrm{~nm}, \gamma=21.7 \mathrm{mN} / \mathrm{m}$, and $\theta=5^{\circ}$.

Fig. 5(c) that $\sigma_{\max }=\sigma_{Y}$ at much smaller structural deformations when $\sigma_{Y}=20 \mathrm{MPa}$ rather than $\sigma_{Y}=40 \mathrm{MPa}$, and that this leads to a reduced $H_{c}$ of $599 \mathrm{~nm}$. The $H_{c}$ predicted by the elastoplastic model is $93 \mathrm{~nm}$ or $15.5 \%$ smaller than the elastic model prediction. When $\sigma_{Y}=20 \mathrm{MPa}$ the elastic model introduces substantial deviations from the elastoplastic beam bending model predictions by neglecting the plastically yielding regions of the deforming structure. Furthermore, a critical height of collapse of $555 \mathrm{~nm}$ was predicted by the plastic model for the case of $E=3 \mathrm{GPa}$ and $\sigma_{Y}$ $=20 \mathrm{MPa}$ [see Fig. 5(c)]. Unlike the case of large yield stresses such as $\sigma_{Y}=40 \mathrm{MPa}$, smaller yield stresses cause the plastic model to usually underestimate $H_{c}$.

Figures 6 and 7 contain plots of the critical height of collapse as a function of structure separation and structure width, respectively. In each figure $H_{c}$ is calculated for (a) $E=3 \mathrm{GPa}$ and $\sigma_{Y}=40 \mathrm{MPa}$ and (b) $E=3 \mathrm{GPa}$ and $\sigma_{Y}$ $=20 \mathrm{MPa}$. In Figs. 6(a) and 7(a) the elastic and elastoplastic models predict nearly identical collapse behavior, while in Figs. 6(b) and 7(b) the two models differ significantly. Note that the elastic model results do not vary between cases (a) and (b) because the yield stress and not the Young's modulus is modified. Further analysis of Fig. 6 indicates that the plastic model calculations approach those of the elastoplastic model at large initial capillary forces (small $d$ ) regardless of the material's yield stress. When the initial capillary forces acting on the beams are very large, the stresses throughout the beams become greater than the yield stress of the material and the entire beams immediately undergo plastic deformation. In addition, for extremely narrow beams (small $W$ in Fig. 7) the plastic model again approaches the elastoplastic model. This result validates the application of the plastic model by Namatsu et al. to Si beams 10 to $50 \mathrm{~nm}$ wide. ${ }^{7}$

The deformation results presented in Figs. 5-7 indicate that there are regimes in $E$ and $\sigma_{Y}$ space for which the elastic and elastoplastic models are equivalent, as well as that there are other regimes for which the models predict significantly different deformation behavior. Although the prior analysis has been performed for mechanical properties typical of polymer materials, the elastoplastic beam bending model and
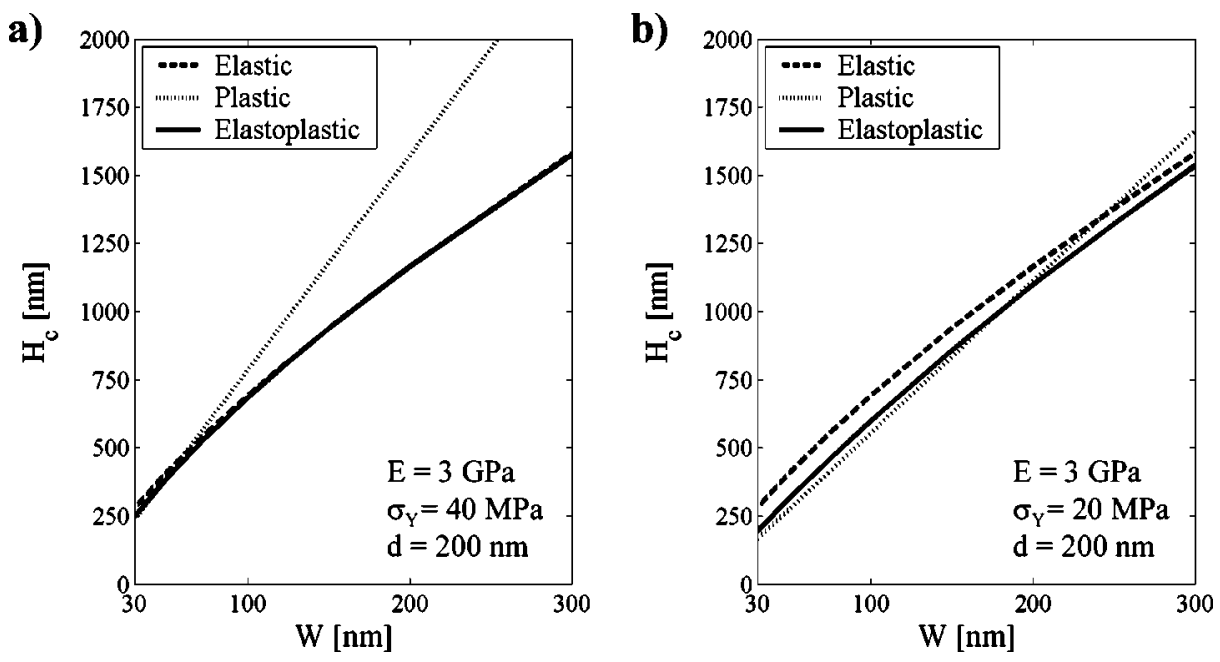

FIG. 7. Comparison between the $H_{c}$ predicted by the elastic, plastic, and elastoplastic models as a function of beam width $W$. (a) The elastic and elastoplastic models estimate nearly identical deformation behavior when $E=3 \mathrm{GPa}$ and $\sigma_{Y}=40 \mathrm{MPa}$, but in the case of (b) $E=3 \mathrm{GPa}$ and a lower yield stress, $\sigma_{Y}=20 \mathrm{MPa}$, the model predictions deviate significantly. Both plots utilize $d=200 \mathrm{~nm}, \quad \gamma=21.7 \mathrm{mN} / \mathrm{m}$, and $\theta=5^{\circ}$. 


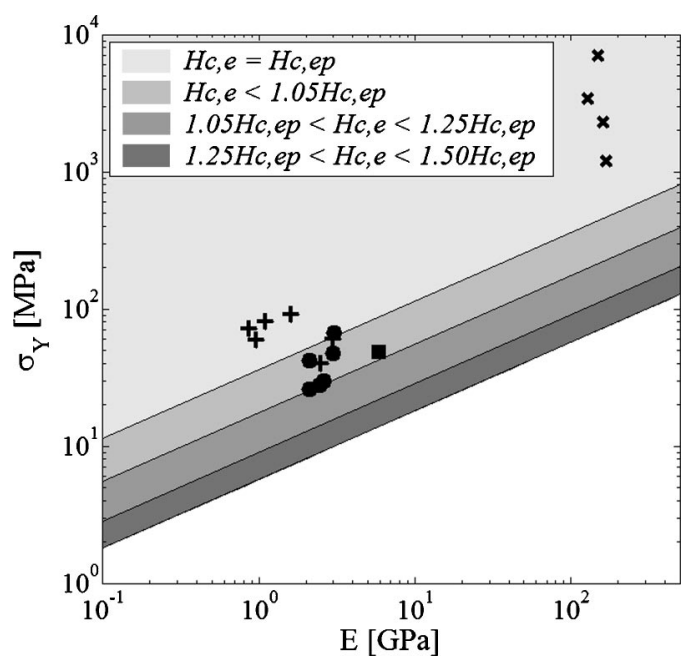

FIG. 8. Regimes in which the elastic and elastoplastic models predict similar collapse behavior. The elastoplastic beam bending model can be applied to materials having any combination of $E$ and $\sigma_{Y}$. However, the elastic model predicts exactly the same critical height of collapse as the elastoplastic model only for materials having a large $\sigma_{Y}$ (shaded region denoted by $\left.H_{c, e}=H_{c, \text { ep }}\right)$. The shaded region $H_{c, e}<1.05 H_{c, \text { ep }}$ encloses the sets of $E$ and $\sigma_{Y}$ for which the critical height of collapse calculated by the elastic model differs by less than $5 \%$ from the predictions of the elastoplastic model. The regions $1.05 H_{c \text {, ep }}<H_{c, e}<1.25 H_{c \text {,ep }}$ and $1.25 H_{c, \text { ep }}<H_{c, e}<1.50 H_{c \text {,ep }}$ enclose the $E-\sigma_{Y}$ space in which the elastic model predicts the critical height to within $25 \%$ and $50 \%$, respectively, of the elastoplastic result. In addition, typical literature values for the $E$ and $\sigma_{Y}$ of PMMA (+), PS ( ), novolak (घ), and $\mathrm{Si}(\times)$ are included. These data were compiled for the case of $d$ $=200 \mathrm{~nm}, W=100 \mathrm{~nm}, \gamma=21.7 \mathrm{mN} / \mathrm{m}$, and $\theta=5^{\circ}$.

the developed methodologies can be generalized to other materials. Figure 8 outlines the combinations of Young's modulus and yield stress for which the deviations between the elastoplastic and elastic model predictions become significant. It has been shown that the elastic model remains valid for materials with yield stresses larger than the maximum stress experienced within the structure. The maximum stress of the elastic model at the critical height, $\sigma_{\max , c}$, can be obtained from Eqs. (7), (10), and (12),

$$
\sigma_{\max , c}=\frac{W}{2 I} \frac{H_{c}^{2}}{2} \frac{8 E I}{H_{c}^{2}} \sqrt{\frac{3 \gamma \cos \theta}{2 E W^{3}}}=\sqrt{\frac{6 E \gamma \cos \theta}{W}} .
$$

The boundary between the elastic and elastoplastic model occurs when $\sigma_{\max , c}=\sigma_{Y}$ and when $\sigma_{\max , c} \leqslant \sigma_{Y}$ the elastic model exactly describes the structural deformation. There are also substantial regimes in $E-\sigma_{Y}$ space in which the results of the elastic model deviate by less than 5\%, 25\%, and 50\% from the elastoplastic model (see Fig. 8). Although the elastic and elastoplastic models become equivalent for materials with high yield stresses, it is clear that the elastoplastic model must be employed for materials with low yield stresses. Typical literature values for the Young's modulus and yield stress of bulk poly(methyl methacrylate) (PMMA), ${ }^{10-13}$ polystyrene (PS), ${ }^{12,14-16}$ a novolak resin, ${ }^{4,16}$ and Si (Refs. 17-19) are also included in Fig. 8. It is important to note that there are a number of $E-\sigma_{Y}$ data points for each type of material; measured mechanical properties are often dependent upon the experimental testing conditions, the characterization temperature, and the processing history of the sample. It can be concluded from Fig. 8 that the col- lapse of PMMA, PS, novolak, and Si beams can be successfully described, to within $\sim 5 \%$ accuracy, by the elastoplastic and elastic models of deformation. Both models can be applied if the error associated with the experimental deformation measurements is greater than the deviation between the elastoplastic and elastic model predictions. For example, Tanaka et al. utilized the elastic model to calculate a Young's modulus of $E=5.4 \mathrm{GPa}$ for a novolak-based photoresist. ${ }^{4}$ If the yield stress of the material was greater than $83.7 \mathrm{MPa}$, then the elastic and elastoplastic model predictions are equivalent. The elastic model can also be applied for novolak materials with $\sigma_{Y}>40.4 \mathrm{MPa}$ as long as a difference of 5\% between the model calculations is acceptable.

There are many materials that could potentially require the application of the elastoplastic model. For example, commercially available chemically amplified photoresists are expected to have lower Young's moduli and yield stresses than pure polymers, such as PMMA and PS, as a result of the presence of photoacid generators, residual casting solvent, and other small molecule components that may act as plasticizers. In addition, polymer mechanical properties are often characterized by significant temperature dependence. At low temperatures pure polymers are brittle materials with high Young's moduli, but at high temperatures they are tough, rubbery materials that can undergo substantial plastic deformation. ${ }^{12}$ Depending on the polymer processing temperatures it may be necessary to utilize the elastoplastic model rather than the elastic model. Furthermore, industrial applications that utilize lithographically defined photoresist nanostructures as an etch mask demand precise control over the feature height and aspect ratio. Pattern collapse is very sensitive to the aspect ratio of the feature and the onset of collapse can occur for increases in height much smaller than $5 \%$. In these types of situations it is important to be able to predict deformation behavior as accurately as possible and the model based on elastoplastic mechanical properties must be applied. Finally, the introduced methodologies could be important for classes of materials other than polymers (e.g., nanostructured $\mathrm{Si}$ as studied by Namatsu et al..$^{7}$ ). The model that includes elastoplastic properties could be critical for calculating the deformation behavior of beams of highly ductile metals such as aluminum and aluminum alloys $(E=70 \mathrm{GPa}$ and $\left.\sigma_{Y}=100-300 \mathrm{MPa}\right){ }^{9}$ In the semiconductor industry these types of materials are commonly used as the interconnect conductors in integrated circuits.

\section{CONCLUSIONS}

Microstructures and nanostructures of polymeric photoresist are deformed and collapse in response to significant capillary forces present during the standard lithographic process. A continuum-level beam bending model that incorporates the material's elastoplastic mechanical properties has been developed and can be used to study the deformation behavior of photoresist structures. As has been demonstrated by a simple example, it is possible to calculate the critical height of collapse based on the mechanical properties of the material and the geometric dimensions of the structure. The elastoplastic beam bending model has been compared to both 
purely elastic and purely plastic models, and generalized regimes in Young's modulus-yield stress space for which it is necessary to use the developed model have been outlined. It is critical to consider both the elastic and plastic mechanical properties when modeling the deformation behavior of materials having low yield stresses. When combined with suitable experimental test structures, the developed elastoplastic beam bending model should provide a powerful technique for measuring the mechanical properties of microstructured and nanostructured polymers.

\section{ACKNOWLEDGMENTS}

This work was supported by the Semiconductor Research Corporation (Grant No. 2002-MJ-985), the NSF Nanoscale Interdisciplinary Research Team program (Grant No. CTS-0210588), Sematech, and Intel Corporation. M.P.S. would like to thank the Graduate Fellowship Program of the Semiconductor Research Corporation.

${ }^{1}$ International SEMATECH, The International Technology Roadmap for Semiconductors: 2002 Update.

${ }^{2}$ L. F. Thompson, C. G. Willson, and M. J. Bowden, Introduction to Microlithography, 2nd ed. (American Chemical Society, Washington, D.C., 1994).

${ }^{3}$ T. Tanaka, M. Morigami, and N. Atoda, J. Electrochem. Soc. 140, L115 (1993).
${ }^{4}$ T. Tanaka, M. Morigami, and N. Atoda, Jpn. J. Appl. Phys., Part 1 32, 6059 (1993).

${ }^{5}$ H. B. Cao, P. F. Nealey, and W. D. Domke, J. Vac. Sci. Technol. B 18, 3303 (2000).

${ }^{6}$ M. P. Stoykovich, H. B. Cao, K. Yoshimoto, L. E. Ocola, and P. F. Nealey, Adv. Mater. (Weinheim, Ger.) 15, 1180 (2003).

${ }^{7}$ H. Namatsu, K. Kurihara, M. Nagase, K. Iwadate, and K. Murase, Appl. Phys. Lett. 66, 2655 (1995).

${ }^{8}$ A. W. Adamson and A. P. Gast, Physical Chemistry of Surfaces, 6th ed. (Wiley, New York 1997).

${ }^{9}$ F. P. Beer, E. R. Johnston, Jr., and J. T. DeWolf, Mechanics of Materials, 3rd ed. (McGraw-Hill, New York, 2001).

${ }^{10}$ M. E. James, Polymer Data Handbook (Oxford University Press, New York, 1999).

${ }^{11}$ R. S. Lakes, Viscoelastic Solids (CRC, Boca Raton, FL 1999).

${ }^{12}$ J. M. G. Cowie, Polymers: Chemistry and Physics of Modern Materials, 2nd ed. (Chapman and Hall, Great Britain, 1991).

${ }^{13}$ W. M. Cheng, G. A. Miller, J. A. Manson, R. W. Hertzberg, and L. H. Sperling, J. Mater. Sci. 25, 1917 (1990).

${ }^{14}$ D. Halliday, R. Resnick, and J. Walker, Fundamentals of Physics, 5th ed. (Wiley, New York, 1997).

${ }^{15}$ I. M. Ward, Mechanical Properties of Solid Polymers, 2nd ed. (Wiley, New York, 1983).

${ }^{16}$ I. I. Rubin, Handbook of Plastic Materials and Technology (Wiley, New York, 1990).

${ }^{17}$ W. N. Sharpe Jr., B. Yuan, R. L. Edwards, and R. Vaidyanathan, in Proceedings of the 10th IEEE International Workshop on Microelectromechanical Systems (Nagoya, Japan, 1997), pp. 424-429.

${ }^{18}$ C. Wilson and P. Beck, J. Microelectromech. Syst. 5, 142 (1996).

${ }^{19}$ K. Petersen, Proc. IEEE 70, 420 (1982). 